\title{
Existence and Ulam-Hyers stability of coupled sequential fractional differential equations with integral boundary conditions
}

\section{Nazim I. Mahmudov ${ }^{1 *}$ and Areen Al-Khateeb ${ }^{1}$}

"Correspondence:

nazim.mahmudov@emu.edu.tr

1 Department of Mathematics,

Eastern Mediterranean University,

Gazimagusa TRNC, Turkey

\begin{abstract}
We study the existence and uniqueness of solutions for coupled sequential fractional differential equations involving Caputo fractional derivative of order $1<\alpha \leq 2$ with integral boundary conditions. Moreover, we discuss Ulam-Hyers stability for the problem at hand.
\end{abstract}

MSC: $34 \mathrm{~A} 08 ; 34 \mathrm{~B}$

Keywords: Fractional differential equations; Mixed boundary value problem; Fixed point theorem

\section{Introduction}

The theory of differential equations of fractional order, involving various types of boundary conditions, has been the subject of interest in the pure and applied sciences. In addition to the classical two-point boundary conditions, much attention is paid to non-local multipoint and integral boundary conditions. Non-local conditions are used to describe certain features of physical, chemical, or other processes occurring in the inner positions of a given region, while integral boundary conditions provide a plausible and practical approach to modeling blood flow problems. For more information and explanation, see, for example, $[1,2]$. Some recent results on the boundary value problem of fractional order can be found in the series [3-21] and in the references cited there. Sequential differential equations with fractional derivatives also received considerable attention, for example, see [4-9].

The study of coupled systems involving fractional differential equations is also important because these systems occur in various problems of applied nature. Coupled systems of fractional differential equations have also been investigated by many authors. Such systems appear naturally in many real world situations. Some recent results on the topic can be found in $[7,17,22-26]$.

(c) The Author(s) 2019. This article is distributed under the terms of the Creative Commons Attribution 4.0 International License (http://creativecommons.org/licenses/by/4.0/), which permits unrestricted use, distribution, and reproduction in any medium, provided you give appropriate credit to the original author(s) and the source, provide a link to the Creative Commons license, and indicate if changes were made. 
We study the following nonlinear sequential fractional differential equation subject to nonseparated nonlocal integral fractional boundary conditions:

$$
\left\{\begin{array}{lc}
\left(D^{\alpha}+\lambda D^{\alpha-1}\right) x(t)=f_{1}(t, x(t), y(t)), & 1<\alpha \leq 2,0 \leq t \leq T, \\
\left(D^{\beta}+\lambda D^{\beta-1}\right) y(t)=f_{2}(t, x(t), y(t)), & 1<\beta \leq 2,0 \leq t \leq T, \\
\nu_{1} x(\eta)+\mu_{1} x(T)=\int_{0}^{T} h_{1}(x(s)) d s, & \nu_{1} y(\eta)+\mu_{1} y(T)=\int_{0}^{T} h_{2}(y(s)) d s, \\
\nu_{2} x^{\prime}(\eta)+\mu_{2} x^{\prime}(T)=\int_{0}^{T} g_{1}(x(s),) d s, & \nu_{2} y^{\prime}(\eta)+\mu_{2} y^{\prime}(T)=\int_{0}^{T} g_{2}(y(s)) d s,
\end{array}\right.
$$

where $D^{\alpha}, D^{\beta}$ denote the Caputo derivative, $0<\eta<T, \lambda \in \mathbb{R}_{+}, \nu_{1}, \nu_{2}, \mu_{1}, \mu_{2} \in \mathbb{R}$.

During the last few decades another part of research, which has been considered for fractional differential equations and got much attention from the researchers, is stability analysis. Numerous forms of stabilities have been studied in literature which are Mittag-Leffler stability, exponential stability, Lyapunov stability, etc. For historical background of UlamHyers stability and recent results, we refer to works [27-36]. To the best of our knowledge, the Ulam-Hyers stability has been very rarely studied for coupled system of fractional differential equations. Therefore in this article we investigate existence and Ulam-Hyers stability to the considered problem. The rest of the paper is organized as follows. In Sect. 2, we recall some basic concepts of fractional calculus and obtain the integral solution for the linear variants of the given problems. Section 3 contains the existence results for problem (1.1) obtained by applying Leray-Schauder's nonlinear alternative, Banach's contraction mapping principle. In Sect. 4, Ulam-Hyers stability for problem (1.1) is studied. Finally, in Sect. 5, an example is provided to illustrate the theoretical results.

\section{Preliminaries}

We begin this section with some basic definitions of fractional calculus [2]. Later we prove an auxiliary lemma, which plays a key role in defining a fixed point problem associated with the given problem.

Definition 1 The Riemann-Liouville fractional integral of order $\alpha>0$ for a function $f$ : $[0,+\infty) \rightarrow R$ is defined as

$$
I_{0+}^{\alpha} f(t)=\frac{1}{\Gamma(\alpha)} \int_{0}^{t}(t-s)^{\alpha-1} f(s) d s
$$

provided that the right-hand side of the integral is pointwise defined on $(0,+\infty)$ and $\Gamma$ is the gamma function.

Definition 2 The Caputo derivative of order $\alpha>0$ for a function $f:[0,+\infty) \rightarrow R$ is written as

$$
D_{0+}^{\alpha} f(t)=\frac{1}{\Gamma(n-\alpha)} \int_{0}^{t}(t-s)^{n-\alpha-1} f^{(n)}(s) d s,
$$

where $n=[\alpha]+1,[\alpha]$ is an integral part of $\alpha$.

Lemma 1 ([2]) Let $\alpha>0$. Then the differential equation $D_{0+}^{\alpha} f(t)=0$ has solutions

$$
f(t)=c_{0}+c_{1} t+c_{2} t^{2}+\cdots+c_{n-1} t^{n-1},
$$


and

$$
I_{0+}^{\alpha} D_{0+}^{\alpha} f(t)=f(t)+c_{0}+c_{1} t+c_{2} t^{2}+\cdots+c_{n-1} t^{n-1},
$$

where $c_{i} \in \mathbb{R}$ and $i=1,2, \ldots, n=[\alpha]+1$.

Let $C([0, T] ; \mathbb{R})$ denote the Banach space of all continuous functions from $[0, T]$ to $\mathbb{R}$ equipped with the sup-norm $\|x\|_{\infty}=\sup \{|x(t)|: 0 \leq t \leq T\}$. For computational convenience, in what follows we use the following notations:

$$
\begin{aligned}
& a_{11}:=v_{1} e^{-\lambda \eta}+\mu_{1} e^{-\lambda T}, \quad a_{12}:=v_{1} \frac{1}{\lambda}\left(1-e^{-\lambda \eta}\right)+\mu_{1} \frac{1}{\lambda}\left(1-e^{-\lambda T}\right), \\
& a_{21}:=-\lambda \nu_{2} e^{-\lambda \eta}-\lambda \mu_{2} e^{-\lambda T}, \quad a_{22}:=\nu_{2} e^{-\lambda \eta}+\mu_{2} e^{-\lambda T}, \\
& \Delta:=a_{11} a_{22}-a_{12} a_{21}, \quad \Delta \neq 0, \\
& \varphi_{1}(t)=\left(\frac{a_{12}}{\Delta} e^{-\lambda t}-\frac{a_{11}}{\Delta} \frac{1}{\lambda}\left(1-e^{-\lambda t}\right)\right), \\
& \varphi_{2}(t)=\left(\frac{a_{21}}{\Delta} \frac{1}{\lambda}\left(1-e^{-\lambda t}\right)-\frac{a_{22}}{\Delta} e^{-\lambda t}\right) .
\end{aligned}
$$

Lemma 2 Let $\rho, \gamma_{1}, \gamma_{2} \in C([0, T] ; \mathbb{R})$. Then the following boundary value problem

$$
\left\{\begin{array}{l}
\left(D^{\alpha}+\lambda D^{\alpha-1}\right) z(t)=\rho(t), \quad 1<\alpha \leq 2,0 \leq t \leq T \\
v_{1} z(\eta)+\mu_{1} z(T)=\int_{0}^{T} \gamma_{1}(s) d s \\
v_{2} z^{\prime}(\eta)+\mu_{2} z^{\prime}(T)=\int_{0}^{T} \gamma_{2}(s) d s
\end{array}\right.
$$

is equivalent to the fractional integral equation

$$
\begin{aligned}
z(t)= & \int_{0}^{t}(t-s)^{\alpha-1} E_{1, \alpha}(-\lambda ; t-s) \rho(s) d s \\
& +\sum_{j=1}^{2} v_{j} \varphi_{j}(t) \int_{0}^{\eta}(\eta-s)^{\alpha-j} E_{1, \alpha+j-1}(-\lambda ; \eta-s) \rho(s) d s \\
& +\sum_{j=1}^{2} \mu_{j} \varphi_{j}(t) \int_{0}^{T}(T-s)^{\alpha-j} E_{1, \alpha+j-1}(-\lambda ; T-s) \rho(s) d s \\
& -\sum_{j=1}^{2} \varphi_{i}(t) \int_{0}^{T} \gamma_{i}(s) d s .
\end{aligned}
$$

Proof Applying $I^{\alpha-1}$ to both sides of (2.2) and using (2.1), we get

$$
\begin{aligned}
& I^{\alpha-1} D^{\alpha-1}(D+\lambda) z(t)=I^{\alpha-1} \rho(t), \\
& (D+\lambda) z(t)=c_{0}+I^{\alpha-1} \rho(t) .
\end{aligned}
$$


We solve the above linear ordinary differential equation:

$$
\begin{aligned}
z(t) & =c_{1} e^{-\lambda t}+c_{0} \frac{1}{\lambda}-c_{0} \frac{1}{\lambda} e^{-\lambda t}+\int_{0}^{t} e^{-\lambda(t-s)} I^{\alpha-1} \rho(s) d s \\
& =c_{1} e^{-\lambda t}+c_{0} \frac{1}{\lambda}\left(1-e^{-\lambda t}\right)+\int_{0}^{t}(t-r)^{\alpha-1} E_{1, \alpha}(-\lambda ; t-r) \rho(r) d r .
\end{aligned}
$$

It is clear that

$$
z^{\prime}(t)=-\lambda c_{1} e^{-\lambda t}+c_{0} e^{-\lambda t}+\int_{0}^{t}(t-r)^{\alpha-2} E_{1, \alpha-1}(-\lambda ; t-r) \rho(r) d r .
$$

The first boundary condition implies that

$$
\begin{aligned}
v_{1} z(\eta)+\mu_{1} z(T) & \\
= & v_{1} c_{1} e^{-\lambda \eta}+v_{1} c_{0} \frac{1}{\lambda}\left(1-e^{-\lambda \eta}\right)+v_{1} \int_{0}^{\eta}(\eta-r)^{\alpha-1} E_{1, \alpha}(-\lambda ; \eta-r) \rho(r) d r \\
& +\mu_{1} c_{1} e^{-\lambda T}+\mu_{1} c_{0} \frac{1}{\lambda}\left(1-e^{-\lambda T}\right)+\mu_{1} \int_{0}^{T}(T-r)^{\alpha-1} E_{1, \alpha}(-\lambda ; T-r) \rho(r) d r \\
= & \int_{0}^{T} \gamma_{1}(s) d s .
\end{aligned}
$$

It follows that

$$
\begin{gathered}
\left(v_{1} e^{-\lambda \eta}+\mu_{1} e^{-\lambda T}\right) c_{1}+\left(v_{1} \frac{1}{\lambda}\left(1-e^{-\lambda \eta}\right)+\mu_{1} \frac{1}{\lambda}\left(1-e^{-\lambda T}\right)\right) c_{0} \\
=\int_{0}^{T} \gamma_{1}(s) d s-v_{1} \int_{0}^{\eta}(\eta-r)^{\alpha-1} E_{1, \alpha}(-\lambda ; \eta-r) \rho(r) d r \\
\quad-\mu_{1} \int_{0}^{T}(T-r)^{\alpha-1} E_{1, \alpha}(-\lambda ; T-r) \rho(r) d r
\end{gathered}
$$

The second boundary condition with (2.5) implies that

$$
\begin{aligned}
& v_{2} z^{\prime}(\eta)+\mu_{2} z^{\prime}(T) \\
& =v_{2}\left(-\lambda c_{1} e^{-\lambda \eta}+c_{0} e^{-\lambda \eta}\right)+v_{2} \int_{0}^{\eta}(\eta-r)^{\alpha-2} E_{1, \alpha-1}(-\lambda ; \eta-r) \rho(r) d r \\
& \quad+\mu_{2}\left(-\lambda c_{1} e^{-\lambda T}+c_{0} e^{-\lambda T}\right)+\mu_{2} \int_{0}^{T}(T-r)^{\alpha-2} E_{1, \alpha-1}(-\lambda ; T-r) \rho(r) d r \\
& =\int_{0}^{T} \gamma_{2}(s) d s .
\end{aligned}
$$

Thus

$$
\begin{aligned}
a_{11} c_{1}+a_{12} c_{0}= & \int_{0}^{T} \gamma_{1}(s) d s-v_{1} \int_{0}^{\eta}(\eta-r)^{\alpha-1} E_{1, \alpha}(-\lambda ; \eta-r) \rho(r) d r \\
& -\mu_{1} \int_{0}^{T}(T-r)^{\alpha-1} E_{1, \alpha}(-\lambda ; T-r) \rho(r) d r,
\end{aligned}
$$




$$
\begin{aligned}
a_{21} c_{1}+a_{22} c_{0}= & \int_{0}^{T} \gamma_{2}(s) d s-v_{2} \int_{0}^{\eta}(\eta-r)^{\alpha-2} E_{1, \alpha-1}(-\lambda ; \eta-r) \rho(r) d r \\
& -\mu_{2} \int_{0}^{T}(T-r)^{\alpha-2} E_{1, \alpha-1}(-\lambda ; T-r) \rho(r) d r .
\end{aligned}
$$

Solving the above system of equations for $c_{0}$ and $c_{1}$, we get

$$
\begin{aligned}
c_{0}= & \frac{a_{11}}{\Delta}\left(\int_{0}^{T} \gamma_{2}(s) d s-v_{2} \int_{0}^{\eta}(\eta-r)^{\alpha-2} E_{1, \alpha-1}(-\lambda ; \eta-r) \rho(r) d r\right. \\
& \left.-\mu_{2} \int_{0}^{T}(T-r)^{\alpha-2} E_{1, \alpha-1}(-\lambda ; T-r) \rho(r) d r\right) \\
& -\frac{a_{21}}{\Delta}\left(\int_{0}^{T} \gamma_{1}(s) d s-v_{1} \int_{0}^{\eta}(\eta-r)^{\alpha-1} E_{1, \alpha}(-\lambda ; \eta-r) \rho(r) d r\right. \\
& \left.-\mu_{1} \int_{0}^{T}(T-r)^{\alpha-1} E_{1, \alpha}(-\lambda ; T-r) \rho(r) d r\right), \\
c_{1}= & \frac{a_{22}}{\Delta}\left(\int_{0}^{T} \gamma_{1}(s) d s-v_{1} \int_{0}^{\eta}(\eta-r)^{\alpha-1} E_{1, \alpha}(-\lambda ; \eta-r) \rho(r) d r\right. \\
& \left.-\mu_{1} \int_{0}^{T}(T-r)^{\alpha-1} E_{1, \alpha}(-\lambda ; T-r) \rho(r) d r\right) \\
& -\frac{a_{12}}{\Delta}\left(\int_{0}^{T} \gamma_{2}(s) d s-v_{2} \int_{0}^{\eta}(\eta-r)^{\alpha-2} E_{1, \alpha-1}(-\lambda ; \eta-r) \rho(r) d r\right. \\
& \left.-\mu_{2} \int_{0}^{T}(T-r)^{\alpha-2} E_{1, \alpha-1}(-\lambda ; T-r) \rho(r) d r\right) .
\end{aligned}
$$

Inserting $c_{0}$ and $c_{1}$ in (2.4), we obtain the desired formula (2.3).

Conversely, assume that $u$ satisfies (2.3). By a direct computation, it follows that the solution given by (2.3) satisfies (2.2).

Lemma 3 For any $g, h \in C([0, T] ; \mathbb{R}), \gamma>0$, we have

$$
\left|\int_{0}^{t}(t-s)^{\gamma-1} E_{1, \gamma}(-\lambda ; t-s)(g(s)-h(s)) d s\right| \leq t^{\gamma} E_{1, \gamma+1}(-\lambda ; t)\|g-h\|_{\infty} .
$$

Proof Indeed,

$$
\begin{aligned}
& \left|\int_{0}^{t}(t-s)^{\gamma-1} E_{1, \gamma}(-\lambda ; t-s)(g(s)-h(s)) d s\right| \\
& \quad \leq \sum_{k=0}^{\infty} \frac{\lambda^{k}}{\Gamma(k+\gamma)} \int_{0}^{t}(t-s)^{k+\gamma-1}|g(s)-h(s)| d s \\
& \quad \leq \sum_{k=0}^{\infty} \frac{\lambda^{k} t^{k+\gamma}}{\Gamma(k+\gamma+1)}=t^{\gamma} E_{1, \gamma+1}(|\lambda| ; t)\|g-h\|_{\infty} .
\end{aligned}
$$

\section{Main results}

By Lemma 2, we introduce a fixed point problem associated with the problem as follows:

$$
\begin{aligned}
(x, y) & =\left(T_{1}, T_{2}\right)(x, y)=\mathfrak{T}(x, y): C([0, T] ; \mathbb{R}) \times C([0, T] ; \mathbb{R}) \\
& \rightarrow C([0, T] ; \mathbb{R}) \times C([0, T] ; \mathbb{R}),
\end{aligned}
$$


where

$$
\begin{aligned}
T_{1}(x, y)(t)= & \int_{0}^{t}(t-r)^{\alpha-1} E_{1, \alpha}(-\lambda ; t-r) f_{1}(r, x(r), y(r)) d r \\
& +\sum_{j=1}^{2} \nu_{j} \varphi_{j}(t) \int_{0}^{\eta}(\eta-r)^{\alpha-j} E_{1, \alpha+j-1}(-\lambda ; \eta-r) f_{1}(r, x(r), y(r)) d r \\
& +\sum_{j=1}^{2} \mu_{j} \varphi_{j}(t) \int_{0}^{T}(T-r)^{\alpha-j} E_{1, \alpha+j-1}(-\lambda ; T-r) f_{1}(r, x(r), y(r)) d r \\
& +\sum_{j=1}^{2} \varphi_{i}(t) \int_{0}^{T} h_{i}(r, x(r), y(r)) d r, \\
T_{2}(x, y)(t)= & \int_{0}^{t}(t-r)^{\beta-1} E_{1, \beta}(-\lambda ; t-r) f_{2}(r, x(r), y(r)) d r \\
& +\sum_{j=1}^{2} v_{j} \varphi_{j}(t) \int_{0}^{\eta}(\eta-r)^{\beta-j} E_{1, \beta+j-1}(-\lambda ; \eta-r) f_{2}(r, x(r), y(r)) d r \\
& +\sum_{j=1}^{2} \mu_{j} \varphi_{j}(t) \int_{0}^{T}(T-r)^{\beta-j} E_{1, \beta+j-1}(-\lambda ; T-r) f_{2}(r, x(r), y(r)) d r \\
& +\sum_{j=1}^{2} \varphi_{i}(t) \int_{0}^{T} g_{i}(r, x(r), y(r)) d r .
\end{aligned}
$$

Evidently, the existence of fixed points of the operator $\mathfrak{T}$ is equivalent to the existence of solutions for problem (1.1).

For $\gamma=\alpha, \beta$, let

$$
\begin{aligned}
R^{\gamma}:= & \max \left\{\left(T^{\gamma} E_{1, \gamma+1}(|\lambda| ; T)+\sum_{j=1}^{2}\left|v_{j}\right|\left\|\varphi_{j}\right\| \eta^{\gamma-j} E_{1, \gamma+j-1}(|\lambda| ; \eta)\right.\right. \\
& \left.\left.+\sum_{j=1}^{2}\left|\mu_{j}\right|\left\|\varphi_{j}\right\| T^{\gamma-j} E_{1, \gamma+j-1}(|\lambda| ; T)\right),\left(\left\|\varphi_{1}\right\|+\left\|\varphi_{2}\right\|\right) T\right\} .
\end{aligned}
$$

Here, we prove the existence and uniqueness of solutions for problem (1.1). We apply a fixed point theorem due to Banach.

Theorem 1 Let $f_{i}, h_{i}, g_{i}:[0, T] \times \mathbb{R} \times \mathbb{R} \rightarrow \mathbb{R}$ be continuous functions such that the following conditions hold:

$\left(\mathrm{A}_{1}\right)$ There exist $L_{f}, L_{h}, L_{g}>0$ such that

$$
\begin{aligned}
& \left|f_{i}\left(t, x_{1}, y_{1}\right)-f_{i}\left(t, x_{2}, y_{2}\right)\right| \leq L_{f}\left(\left|x_{1}-x_{2}\right|+\left|y_{1}-y_{2}\right|\right), \\
& \left|h_{i}\left(t, x_{1}, y_{1}\right)-h_{i}\left(t, x_{2}, y_{2}\right)\right| \leq L_{h}\left(\left|x_{1}-x_{2}\right|+\left|y_{1}-y_{2}\right|\right), \\
& \left|g_{i}\left(t, x_{1}, y_{1}\right)-g_{i}\left(t, x_{2}, y_{2}\right)\right| \leq L_{g}\left(\left|x_{1}-x_{2}\right|+\left|y_{1}-y_{2}\right|\right), \\
& \forall\left(t, x_{1}, y_{1}\right),\left(t, x_{2}, y_{2}\right) \in[0, T] \times \mathbb{R} \times \mathbb{R} .
\end{aligned}
$$


$\left(\mathrm{A}_{2}\right) 1-2\left(L_{f}+L_{h}\right) R^{\alpha}>0,1-2\left(L_{f}+L_{g}\right) R^{\beta}>0$.

Then problem $(1.1)$ has a unique solution in $C([0, T], \mathbb{R}) \times C([0, T], \mathbb{R})$.

Proof Consider a ball

$$
B_{r}:=\left\{u \in C([0, T], \mathbb{R}) \times C([0, T], \mathbb{R}):\|u\|_{\infty} \leq r\right\}
$$

with radius

$$
r \geq \max \left\{\frac{\left(M_{f}+M_{h}\right) R^{\alpha}}{1-2\left(L_{f}+L_{h}\right) R^{\alpha}}, \frac{\left(M_{f}+M_{g}\right) R^{\beta}}{1-2\left(L_{f}+L_{g}\right) R^{\beta}}\right\},
$$

where

$$
\begin{aligned}
M_{f} & :=\max \left(\left\|f_{1}(t, 0,0)\right\|_{\infty},\left\|f_{2}(t, 0,0)\right\|_{\infty}\right), \\
M_{h} & :=\max \left(\left\|h_{1}(t, 0,0)\right\|_{\infty},\left\|h_{2}(t, 0,0)\right\|_{\infty}\right), \\
M_{g} & :=\max \left(\left\|g_{1}(t, 0,0)\right\|_{\infty},\left\|g_{2}(t, 0,0)\right\|_{\infty}\right) .
\end{aligned}
$$

It is clear that for all $x, y \in \mathbb{R}$

$$
\begin{aligned}
& \left|f_{i}(t, x, y)\right| \leq L_{f}(|x|+|y|)+M_{f}, \\
& \left|h_{i}(t, x, y)\right| \leq L_{h}(|x|+|y|)+M_{h}, \\
& \left|g_{i}(t, x, y)\right| \leq L_{g}(|x|+|y|)+M_{g} .
\end{aligned}
$$

Using this inequality and Lemma 3, from (3.1) it follows that

$$
\begin{aligned}
& \left|T_{1}(x, y)(t)\right| \\
& \leq t^{\alpha} E_{1, \alpha+1}(|\lambda| ; t)\left\|f_{i}(\cdot, x(\cdot), y(\cdot))\right\|+\sum_{j=1}^{2}\left|v_{j}\right|\left|\varphi_{j}(t)\right| \eta^{\alpha-j} E_{1, \alpha+j-1}(|\lambda| ; \eta)\left\|f_{i}(\cdot, x(\cdot), y(\cdot))\right\| \\
& \quad+\sum_{j=1}^{2}\left|\mu_{j}\right|\left|\varphi_{j}(t)\right| T^{\alpha-j} E_{1, \alpha+j-1}(|\lambda| ; T)\left\|f_{i}(\cdot, x(\cdot), y(\cdot))\right\|_{\infty} \\
& \quad+\sum_{j=1}^{2}\left|\varphi_{i}(t)\right| \int_{0}^{T}\left|h_{i}(r, x(r), y(r))\right| d r \\
& \leq \quad\left(t^{\alpha} E_{1, \alpha+1}(-\lambda ; t)+\sum_{j=1}^{2}\left|v_{j}\right|\left|\varphi_{j}(t)\right| \eta^{\alpha-j} E_{1, \alpha+j-1}(|\lambda| ; \eta)\right. \\
& \left.\quad+\sum_{j=1}^{2}\left|\mu_{j}\right|\left|\varphi_{j}(t)\right| T^{\alpha-j} E_{1, \alpha+j-1}(|\lambda| ; T)\right) \\
& \quad \times\left(L_{f}(|x|+|y|)+M_{f}\right)+\left(\left\|\varphi_{1}\right\|+\left\|\varphi_{2}\right\|\right) T\left(L_{h}(|x|+|y|)+M_{h}\right) \\
& \leq \\
& \left.\quad\left(L_{f}+L_{h}\right) r+M_{f}+M_{h}\right) R^{\alpha} \leq \frac{r}{2} .
\end{aligned}
$$


In the like manner we have

$$
\left|T_{2}(x, y)(t)\right| \leq\left(\left(L_{f}+L_{h}\right) r+M_{f}+M_{h}\right) R^{\beta} \leq \frac{r}{2} .
$$

From (3.3) and (3.4) it follows that $\mathfrak{T} B_{r} \subset B_{r}$. Next, using condition $\left(\mathrm{A}_{1}\right)$, we obtain

$$
\begin{aligned}
\left|T_{1}\left(x_{1}, y_{1}\right)(t)-T_{1}\left(x_{2}, y_{2}\right)(t)\right| & \leq R^{\alpha}\left\|f_{1}\left(\cdot, x_{1}(\cdot), y_{1}(\cdot)\right)-f_{1}\left(\cdot, x_{2}(\cdot), y_{2}(\cdot)\right)\right\|_{\infty} \\
& \leq\left(L_{f}+L_{h}\right) R^{\alpha}\left\|\left(x_{1}, y_{1}\right)-\left(x_{2}, y_{2}\right)\right\|_{\infty} .
\end{aligned}
$$

Similarly,

$$
\begin{aligned}
\left|T_{2}\left(x_{1}, y_{1}\right)(t)-T_{2}\left(x_{2}, y_{2}\right)(t)\right| & \leq R^{\beta}\left\|f_{2}\left(\cdot, x_{1}(\cdot), y_{1}(\cdot)\right)-f_{2}\left(\cdot, x_{2}(\cdot), y_{2}(\cdot)\right)\right\|_{\infty} \\
& \leq\left(L_{f}+L_{g}\right) R^{\beta}\left\|\left(x_{1}, y_{1}\right)-\left(x_{2}, y_{2}\right)\right\|_{\infty} .
\end{aligned}
$$

It follows from (3.5) and (3.6) that

$$
\left\|T\left(x_{1}, y_{1}\right)-T\left(x_{2}, y_{2}\right)\right\| \leq\left[\left(L_{f}+L_{h}\right) R^{\alpha}+\left(L_{f}+L_{g}\right) R^{\beta}\right]\left\|\left(x_{1}, y_{1}\right)-\left(x_{2}, y_{2}\right)\right\|_{\infty} .
$$

By $\left(\mathrm{A}_{2}\right)$ the operator $\mathfrak{T}$ is a contraction. Thus by the Banach fixed point theorem, $\mathfrak{T}$ has a unique fixed point in $C([0, T], \mathbb{R}) \times C([0, T], \mathbb{R})$. This completes the proof.

In the next result, we prove the existence of solutions for problem (1.1) by applying the Leray-Schauder alternative.

Theorem 2 Let $f .[0, T] \times \mathbb{R} \times \mathbb{R} \rightarrow \mathbb{R}$ be a continuous function such that the following condition holds:

$\left(\mathrm{A}_{3}\right)$ There exist $\gamma_{f}, \gamma_{h}, \gamma_{g} \in C\left([0, T], \mathbb{R}_{+}\right)$and a nondecreasing function $\psi: \mathbb{R}_{+} \rightarrow \mathbb{R}_{+}$such that

$$
\begin{aligned}
& \left|f_{i}(t, x, y)\right| \leq \gamma_{f}(t) \psi_{f}(|x|+|y|), \quad \forall(t, x, y) \in[0, T] \times \mathbb{R} \times \mathbb{R} . \\
& \left|h_{i}(t, x, y)\right| \leq \gamma_{h}(t) \psi_{h}(|x|+|y|), \\
& \left|g_{i}(t, x, y)\right| \leq \gamma_{g}(t) \psi_{g}(|x|+|y|), \quad i=1,2 .
\end{aligned}
$$

$\left(\mathrm{A}_{4}\right)$ There exists $M>0$ such that

$$
\frac{M}{\left(\left\|\gamma_{f}\right\|_{\infty} \psi_{f}(M)+\left\|\gamma_{h}\right\|_{\infty} \psi_{h}(M)\right) R^{\alpha}+\left(\left\|\gamma_{f}\right\|_{\infty} \psi_{f}(M)+\left\|\gamma_{g}\right\|_{\infty} \psi_{g}(M)\right) R^{\beta}}>1 .
$$

Then BVP (1.1) has at least one solution.

Proof Step 1: Show that $\mathfrak{T}: C([0, T], \mathbb{R}) \times C([0, T], \mathbb{R}) \rightarrow C([0, T], \mathbb{R}) \times C([0, T], \mathbb{R})$ maps bounded sets into bounded sets and is continuous.

Let $B_{r}$ be a ball in $C([0, T], \mathbb{R}) \times C([0, T], \mathbb{R})$. Then

$$
\left|f_{1}(t, x(t), y(t))\right| \leq\left\|\gamma_{f}\right\| \psi_{f}(r), \quad\left|f_{2}(t, x(t), y(t))\right| \leq\left\|\gamma_{f}\right\| \psi_{f}(r),
$$


and by Lemma 3

$$
\begin{aligned}
\left|T_{1}(x, y)(t)\right| & \\
\leq & t^{\alpha} E_{1, \alpha+1}(|\lambda| ; t)\left\|f_{1}(\cdot, x(\cdot), y(\cdot))\right\|+\sum_{j=1}^{2}\left|v_{j}\right|\left|\varphi_{j}(t)\right| \eta^{\alpha-j} E_{1, \alpha+j-1}(|\lambda| ; \eta)\left\|f_{i}(\cdot, x(\cdot), y(\cdot))\right\| \\
& +\sum_{j=1}^{2}\left|\mu_{j}\right|\left|\varphi_{j}(t)\right| T^{\alpha-j} E_{1, \alpha+j-1}(|\lambda| ; T)\left\|f_{i}(\cdot, x(\cdot), y(\cdot))\right\|_{\infty} \\
& +\sum_{j=1}^{2}\left|\varphi_{i}(t)\right| \int_{0}^{T}\left|h_{i}(r, x(r), y(r))\right| d r \\
\leq & \left(t^{\alpha} E_{1, \alpha+1}(|\lambda| ; t)+\sum_{j=1}^{2}\left|v_{j}\right|\left|\varphi_{j}(t)\right| \eta^{\alpha-j} E_{1, \alpha+j-1}(|\lambda| ; \eta)\right. \\
& \left.+\sum_{j=1}^{2}\left|\mu_{j}\right|\left|\varphi_{j}(t)\right| T^{\alpha-j} E_{1, \alpha+j-1}(|\lambda| ; T)\right) \\
& \times\left(\gamma_{f}(t) \psi_{f}(|x|+|y|)+M_{f}\right)+\left(\left\|\varphi_{1}\right\|+\left\|\varphi_{2}\right\|\right) T\left(L_{h}(|x|+|y|)+M_{h}\right) \\
\leq & \left(\left\|\gamma_{f}\right\| \psi_{f}(\|(x, y)\|)+\left\|\gamma_{h}\right\| \psi_{h}(\|(x, y)\|)\right) R^{\alpha} .
\end{aligned}
$$

Similarly,

$$
\left|T_{2}(x, y)(t)\right| \leq\left(\left\|\gamma_{f}\right\| \psi_{f}(\|(x, y)\|)+\left\|\gamma_{g}\right\| \psi_{g}(\|(x, y)\|)\right) R^{\beta} .
$$

It follows that $\mathfrak{T}\left(B_{r}\right)$ is bounded.

Step 2: Next we show that $\mathfrak{T}$ maps bounded sets into equicontinuous sets of $C([0, T], \mathbb{R})$. Let $t_{1}, t_{2} \in[0, T]$ with $t_{1}<t_{2}$ and $(x, y) \in B_{r}$. Then we obtain

$$
\begin{aligned}
& \left|T_{1}(x, y)\left(t_{1}\right)-T_{1}(x, y)\left(t_{2}\right)\right| \\
& \leq \mid \int_{0}^{t_{1}}\left(t_{1}-r\right)^{\alpha-1} E_{1, \alpha}\left(-\lambda ; t_{1}-r\right) f_{1}(r, x(r), y(r)) d r \\
& \quad-\int_{0}^{t_{2}}\left(t_{2}-r\right)^{\alpha-1} E_{1, \alpha}\left(-\lambda ; t_{2}-r\right) f_{1}(r, x(r), y(r)) d r \mid \\
& \quad+\sum_{j=1}^{2}\left|v_{j}\right|\left|\varphi_{j}\left(t_{1}\right)-\varphi_{j}\left(t_{2}\right)\right| \eta^{\alpha-j} E_{1, \alpha+j-1}(|\lambda| ; \eta)\left\|f_{i}(\cdot, x(\cdot), y(\cdot))\right\| \\
& \quad+\sum_{j=1}^{2}\left|\mu_{j}\right|\left|\varphi_{j}\left(t_{1}\right)-\varphi_{j}\left(t_{2}\right)\right| T^{\alpha-j} E_{1, \alpha+j-1}(|\lambda| ; T)\left\|f_{i}(\cdot, x(\cdot), y(\cdot))\right\|_{\infty} \\
& \quad+\sum_{j=1}^{2}\left|\varphi_{j}\left(t_{1}\right)-\varphi_{j}\left(t_{2}\right)\right| \int_{0}^{T}\left|h_{i}(r, x(r), y(r))\right| d r .
\end{aligned}
$$


It is clear that the last three terms approach to zero independently of $(x, y) \in B_{r}$ as $t_{1} \rightarrow t_{2}$. Now, we estimate the first term of (3.7):

$$
\begin{aligned}
\mid \int_{0}^{t_{1}}\left(t_{1}-r\right)^{\alpha-1} E_{1, \alpha}\left(-\lambda ; t_{1}-r\right) f_{1}(r, x(r), y(r)) d r \\
\quad-\int_{0}^{t_{2}}\left(t_{2}-r\right)^{\alpha-1} E_{1, \alpha}\left(-\lambda ; t_{2}-r\right) f_{1}(r, x(r), y(r)) d r \mid \\
=\left|\int_{0}^{t_{1}} e^{-\lambda\left(t_{1}-s\right)}\left(I^{\alpha-1} f_{1}(\cdot, x(\cdot), y(\cdot))\right)(s) d s-\int_{0}^{t_{2}} e^{-\lambda\left(t_{2}-s\right)}\left(I^{\alpha-1} f_{1}(\cdot, x(\cdot), y(\cdot))\right)(s) d s\right| \\
\quad \leq\left|\int_{t_{1}}^{t_{2}} e^{-\lambda\left(t_{1}-s\right)}\left(I^{\alpha-1} f_{1}(\cdot, x(\cdot), y(\cdot))\right)(s) d s\right| \\
\quad+\left|\int_{0}^{t_{1}}\left[e^{-\lambda\left(t_{2}-s\right)}-e^{-\lambda\left(t_{1}-s\right)}\right]\left(I^{\alpha-1} f_{1}(\cdot, x(\cdot), y(\cdot))\right)(s) d s\right| .
\end{aligned}
$$

Obviously, the right-hand side of the above inequality tends to zero independently of $(x, y) \in B_{r}$ as $t_{1} \rightarrow t_{2}$. A similar result is true for $T_{1}(x, y)$. As $\mathfrak{T}$ is uniformly bounded and equicontinuous, therefore it follows by the Arzelá-Ascoli theorem that $\mathfrak{T}: C([0, T], \mathbb{R}) \times$ $C([0, T], \mathbb{R}) \rightarrow C([0, T], \mathbb{R}) \times C([0, T], \mathbb{R})$ is completely continuous.

The result will follow from the Leray-Schauder nonlinear alternative once we have proved the boundedness of the set of all solutions to equations $(x, y)=\theta \mathfrak{T}(x, y)$ for $0 \leq$ $\theta \leq 1$.

Let $(x, y)$ be a solution. Then, using the computations employed in proving that $\mathfrak{T}$ is bounded, we have

$$
\begin{aligned}
|(x, y)(t)|= & \theta|\mathfrak{T}(x, y)(t)| \\
\leq & \left(\left\|\gamma_{f}\right\| \psi_{f}(\|(x, y)\|)+\left\|\gamma_{h}\right\| \psi_{h}(\|(x, y)\|)\right) R^{\alpha} \\
& +\left(\left\|\gamma_{f}\right\| \psi_{f}(\|(x, y)\|)+\left\|\gamma_{g}\right\| \psi_{g}(\|(x, y)\|)\right) R^{\beta} .
\end{aligned}
$$

Consequently, we have

$$
\begin{aligned}
& \|(x, y)\|_{\infty} \\
& \quad /\left(\left(\left\|\gamma_{f}\right\|_{\infty} \psi_{f}\left(\|(x, y)\|_{\infty}\right)+\left\|\gamma_{h}\right\|_{\infty} \psi_{h}\left(\|(x, y)\|_{\infty}\right)\right) R^{\alpha}\right. \\
& \left.\quad+\left(\left\|\gamma_{f}\right\|_{\infty} \psi_{f}\left(\|(x, y)\|_{\infty}\right)+\left\|\gamma_{g}\right\|_{\infty} \psi_{g}\left(\|(x, y)\|_{\infty}\right)\right) R^{\beta}\right) \leq 1 .
\end{aligned}
$$

In view of $\left(\mathrm{A}_{4}\right)$, there exists $M$ such that $\|(x, y)\|_{\infty} \neq M$. Let us set

$$
\mathfrak{U}=\left\{(x, y) \in C([0, T], \mathbb{R}) \times C([0, T], \mathbb{R}):\|(x, y)\|_{\infty}<M\right\} .
$$

Note that the operator $\mathfrak{T}: \overline{\mathfrak{U}} \rightarrow C([0, T], \mathbb{R})$ is continuous and completely continuous. From the choice of $\mathfrak{U}$, there is no $(x, y) \in \partial \mathfrak{U}$ such that $(x, y)=\theta \mathfrak{T}(x, y)$ for some $0<\theta<1$. Consequently, by the nonlinear alternative of Leray-Schauder type, we deduce that $\mathfrak{T}$ has a fixed point $(x, y) \in \overline{\mathfrak{U}}$ which is a solution of problem (1.1). This completes the proof. 


\section{Ulam-Hyers stability}

In this section, we discuss the Ulam-Hyers stability for problem (1.1) by means of integral representation of its solution given by

$$
x(t)=T_{1}(x, y)(t), \quad y(t)=T_{2}(x, y)(t),
$$

where $T_{1}$ and $T_{2}$ are defined by (3.1) and (3.2).

Define the following nonlinear operators $Q_{1}, Q_{2}: C([0, T], \mathbb{R}) \times C([0, T], \mathbb{R}) \rightarrow$ $C([0, T], \mathbb{R})$ :

$$
\begin{aligned}
& Q_{1}(x, y)(t):=\left(D^{\alpha}+\lambda D^{\alpha-1}\right) x(t)-f_{1}(t, x(t), y(t)), \\
& Q_{2}(x, y)(t):=\left(D^{\beta}+\lambda D^{\beta-1}\right) y(t)-f_{2}(t, x(t), y(t)) .
\end{aligned}
$$

For some $\varepsilon_{1}, \varepsilon_{2}>0$, we consider the following inequality:

$$
\left\|Q_{1}(x, y)\right\| \leq \varepsilon_{1}, \quad\left\|Q_{2}(x, y)\right\| \leq \varepsilon_{2} .
$$

Definition 3 The coupled system (1.1) is said to be Ulam-Hyers stable if there exist $V_{1}, V_{2}>0$ such that, for every solution $\left(x^{*}, y^{*}\right) \in C([0, T], \mathbb{R}) \times C([0, T], \mathbb{R})$ of inequality (4.1), there exists a unique solution $(x, y) \in C([0, T], \mathbb{R}) \times C([0, T], \mathbb{R})$ of problem $(1.1)$ with

$$
\left\|(x, y)-\left(x^{*}, y^{*}\right)\right\|_{\infty} \leq V_{1} \varepsilon_{1}+V_{2} \varepsilon_{2} .
$$

Theorem 3 Let the assumptions of Theorem 1 hold. Then problem (1.1) is Ulam-Hyers stable.

Proof Let $(x, y) \in C([0, T], \mathbb{R}) \times C([0, T], \mathbb{R})$ be the solution of problem (1.1) satisfying (3.1) and (3.2). Let $\left(x^{*}, y^{*}\right)$ be any solution satisfying (4.1):

$$
\begin{aligned}
& \left(D^{\alpha}+\lambda D^{\alpha-1}\right) x^{*}(t)=f_{1}\left(t, x^{*}(t), y^{*}(t)\right)+Q_{1}\left(x^{*}, y^{*}\right)(t), \\
& \left(D^{\beta}+\lambda D^{\beta-1}\right) y^{*}(t)=f_{2}\left(t, x^{*}(t), y^{*}(t)\right)+Q_{2}\left(x^{*}, y^{*}\right)(t) .
\end{aligned}
$$

So

$$
\begin{aligned}
x^{*}(t)= & T_{1}\left(x^{*}, y^{*}\right)(t)+\int_{0}^{t}(t-r)^{\alpha-1} E_{1, \alpha}(-\lambda ; t-r) Q_{1}\left(x^{*}, y^{*}\right)(r) d r \\
& +\sum_{j=1}^{2} v_{j} \varphi_{j}(t) \int_{0}^{\eta}(\eta-r)^{\alpha-j} E_{1, \alpha+j-1}(-\lambda ; \eta-r) Q_{1}\left(x^{*}, y^{*}\right)(r) d r \\
& +\sum_{j=1}^{2} \mu_{j} \varphi_{j}(t) \int_{0}^{T}(T-r)^{\alpha-j} E_{1, \alpha+j-1}(-\lambda ; T-r) Q_{1}\left(x^{*}, y^{*}\right)(r) d r .
\end{aligned}
$$

It follows that

$$
\begin{aligned}
& \left|T_{1}\left(x^{*}, y^{*}\right)(t)-x^{*}(t)\right| \\
& \quad \leq \int_{0}^{t}(t-r)^{\alpha-1} E_{1, \alpha}(|\lambda| ; t-r) d r \varepsilon_{1}+\sum_{j=1}^{2}\left|v_{j}\right| \mid \varphi_{j} \| \int_{0}^{\eta}(\eta-r)^{\alpha-j} E_{1, \alpha+j-1}(|\lambda| ; \eta-r) d r \varepsilon_{1}
\end{aligned}
$$




$$
\begin{aligned}
& +\sum_{j=1}^{2}\left|\mu_{j}\right|\left\|\varphi_{j}\right\| \int_{0}^{T}(T-r)^{\alpha-j} E_{1, \alpha+j-1}(|\lambda| ; T-r) d r \varepsilon_{1} \\
\leq & \left(T^{\alpha} E_{1, \alpha+1}(|\lambda| ; T)+\sum_{j=1}^{2}\left|v_{j}\right|\left\|\varphi_{j}\right\| \eta^{\alpha-j} E_{1, \alpha+j-1}(|\lambda| ; \eta)\right. \\
& \left.+\sum_{j=1}^{2}\left|\mu_{j}\right|\left\|\varphi_{j}\right\| T^{\alpha-j} E_{1, \alpha+j-1}(|\lambda| ; T)\right) \varepsilon_{1} \\
= & : U^{\alpha} \varepsilon_{1} .
\end{aligned}
$$

Similarly,

$$
\begin{aligned}
& \left|T_{2}\left(x^{*}, y^{*}\right)(t)-y^{*}(t)\right| \\
& \leq\left(T^{\beta} E_{1, \beta+1}(|\lambda| ; T)+\sum_{j=1}^{2}\left|v_{j}\right|\left\|\varphi_{j}\right\| \eta^{\beta-j} E_{1, \beta+j-1}(|\lambda| ; \eta)\right. \\
& \left.+\sum_{j=1}^{2}\left|\mu_{j}\right|\left\|\varphi_{j}\right\| T^{\beta-j} E_{1, \beta+j-1}(|\lambda| ; T)\right) \varepsilon_{2} \\
& =: U^{\beta} \varepsilon_{1} .
\end{aligned}
$$

Therefore, we deduce by the fixed point property of the operator $\mathfrak{T}$, given by (3.1) and (3.2), that

$$
\begin{aligned}
\left|x(t)-x^{*}(t)\right| & =\left|x(t)-T_{1}\left(x^{*}, y^{*}\right)(t)+T_{1}\left(x^{*}, y^{*}\right)(t)-x^{*}(t)\right| \\
& \leq\left|T_{1}(x, y)(t)-T_{1}\left(x^{*}, y^{*}\right)(t)\right|+\left|T_{1}\left(x^{*}, y^{*}\right)(t)-x^{*}(t)\right| \\
& \leq\left(L_{f}+L_{h}\right) U^{\alpha}\left\|(x, y)-\left(x^{*}, y^{*}\right)\right\|_{\infty}+U^{\alpha} \varepsilon_{1},
\end{aligned}
$$

and similarly

$$
\begin{aligned}
\left|y(t)-y^{*}(t)\right| & =\left|y(t)-T_{2}\left(x^{*}, y^{*}\right)(t)+T_{2}\left(x^{*}, y^{*}\right)(t)-y^{*}(t)\right| \\
& \leq\left(L_{f}+L_{g}\right) U^{\beta}\left\|(x, y)-\left(x^{*}, y^{*}\right)\right\|_{\infty}+U^{\beta} \varepsilon_{2} .
\end{aligned}
$$

From (4.3) and (4.4) it follows that

$$
\left\|(x, y)-\left(x^{*}, y^{*}\right)\right\|_{\infty} \leq\left(\left(L_{f}+L_{h}\right) U^{\alpha}+\left(L_{f}+L_{g}\right) U^{\beta}\right)\left\|(x, y)-\left(x^{*}, y^{*}\right)\right\|_{\infty}+U^{\alpha} \varepsilon_{1}+U^{\beta} \varepsilon_{2},
$$

and

$$
\begin{aligned}
\left\|(x, y)-\left(x^{*}, y^{*}\right)\right\|_{\infty} & \leq \frac{U^{\alpha} \varepsilon_{1}+U^{\beta} \varepsilon_{2}}{1-\left(\left(L_{f}+L_{h}\right) U^{\alpha}+\left(L_{f}+L_{g}\right) U^{\beta}\right)} \\
& =V_{1} \varepsilon_{1}+V_{2} \varepsilon_{2},
\end{aligned}
$$

with

$$
V_{1}=\frac{U^{\alpha}}{1-\left(\left(L_{f}+L_{h}\right) U^{\alpha}+\left(L_{f}+L_{g}\right) U^{\beta}\right)},
$$




$$
V_{2}=\frac{U^{\beta}}{1-\left(\left(L_{f}+L_{h}\right) U^{\alpha}+\left(L_{f}+L_{g}\right) U^{\beta}\right)} .
$$

Thus, problem (1.1) is Ulam-Hyers stable.

\section{Application}

We consider the following fractional order coupled system:

$$
\left\{\begin{array}{l}
\left(D^{\alpha}+\lambda D^{\alpha-1}\right) x(t)=L_{f} \frac{|y(t)|}{1+|y(t)|}, \quad 1<\alpha \leq 2,0 \leq t \leq T, \\
\left(D^{\beta}+\lambda D^{\beta-1}\right) y(t)=L_{f}(\sin x(t)+(\cos t) x(t)), \quad 1<\beta \leq 2,0 \leq t \leq T, \\
v_{1} x(\eta)+\mu_{1} x(T)=L_{h} \int_{0}^{T} \frac{|x(t)|}{11+|x(t)|} d s, \\
v_{1} y(\eta)+\mu_{1} y(T)=L_{h} \int_{0}^{T}(\sin y(t)+\cos y(t)) d s, \\
v_{2} x^{\prime}(\eta)+\mu_{2} x^{\prime}(T)=L_{g} \int_{0}^{T} \frac{|x(t)|}{21+|x(t)|} d s, \\
v_{2} y^{\prime}(\eta)+\mu_{2} y^{\prime}(T)=L_{g} \int_{0}^{T}(\sin y(t)+\cos y(t)) d s .
\end{array}\right.
$$

Here

$$
\begin{aligned}
& f_{1}(t, x, y)=L_{f} \frac{|y|}{1+|y|}, \quad f_{2}(t, x, y)=L_{f}(\sin x+(\cos t) x), \quad h_{1}(x)=L_{h} \frac{|x|}{11+|x|}, \\
& h_{2}(y)=L_{h}(\sin y+\cos y), \quad g_{1}(x)=L_{g} \frac{|x|}{21+|x|}, \quad g_{2}(y)=L_{g}(\sin y+\cos y) .
\end{aligned}
$$

As

$$
\begin{array}{ll}
\left|f_{1}\left(t, x_{1}, y_{1}\right)-f_{1}\left(t, x_{2}, y_{2}\right)\right| \leq L_{f}\left|y_{1}-y_{2}\right|, & \left|f_{2}\left(t, x_{1}, y_{1}\right)-f_{2}\left(t, x_{2}, y_{2}\right)\right| \leq L_{f}\left|x_{1}-x_{2}\right|, \\
\left|h_{1}\left(t, x_{1}\right)-h_{1}\left(t, x_{2}\right)\right| \leq L_{h}\left|x_{1}-x_{2}\right|, & \left|h_{2}\left(t, y_{1}\right)-h_{2}\left(t, y_{2}\right)\right| \leq L_{h}\left|y_{1}-y_{2}\right|, \\
\left|g_{1}\left(t, x_{1}\right)-g_{1}\left(t, x_{2}\right)\right| \leq L_{g}\left|x_{1}-x_{2}\right|, & \left|g_{2}\left(t, y_{1}\right)-g_{2}\left(t, y_{2}\right)\right| \leq L_{g}\left|y_{1}-y_{2}\right|,
\end{array}
$$

therefore $\left(\mathrm{A}_{1}\right)$ is satisfied. It is obvious that $L_{f}, L_{h}, L_{g}>0$ can be chosen so that condition $\left(\mathrm{A}_{2}\right)$ is satisfied. Therefore, coupled system (1.1) has a unique solution and is Ulam-Hyers stable.

\section{Conclusion}

Here we have studied the existence and uniqueness of the solutions as well as the UlamHyers stability for a coupled sequential fractional system with integral boundary conditions. As a future work, one can generalize different concepts of stability and existence results to an impulsive fractional system, a neutral time-delay system/inclusion, and a time-delay system/inclusion with finite delay.

\section{Acknowledgements}

The authors would like to thank the reviewers for their valuable suggestions and comments.

Funding

The research was completed under the research project BAPC-04-19-02 of the Eastern Mediterranean University. 
Authors' contributions

All authors read and approved the final manuscript.

\section{Publisher's Note}

Springer Nature remains neutral with regard to jurisdictional claims in published maps and institutional affiliations.

\section{Received: 21 March 2019 Accepted: 30 May 2019 Published online: 07 June 2019}

\section{References}

1. Podlubny, I.: Fractional Differential Equations. Academic Press, San Diego (1999)

2. Kilbas, A.A., Srivastava, H.M., Trujillo, J.J.: Theory and Applications of Fractional Differential Equations. North-Holland Mathematics Studies, vol. 204. Elsevier, Amsterdam (2006)

3. Agarwal, R.P., O'Regan, D., Hristova, S.: Stability of Caputo fractional differential equations by Lyapunov functions. Appl. Math. 60, 653-676 (2015)

4. Bai, C.: Impulsive periodic boundary value problems for fractional differential equation involving Riemann-Liouville sequential fractional derivative. J. Math. Anal. Appl. 384, 211-231 (2011)

5. Ahmad, B., Nieto, J.J.: Sequential fractional differential equations with three-point boundary conditions. Comput. Math. Appl. 64, 3046-3052 (2012)

6. Ahmad, B., Nieto, J.J.: Boundary value problems for a class of sequential integrodifferential equations of fractional order. J. Funct. Spaces Appl. 2013, Art. ID 149659, 8 pp. (2013)

7. Ahmad, B., Ntouyas, S.K.: Existence results for a coupled system of Caputo type sequential fractional differential equations with nonlocal integral boundary conditions. Appl. Math. Comput. 266, 615-622 (2015)

8. Aqlan, M.H., Alsaedi, A., Ahmad, B., Nieto, J.J.: Existence theory for sequential fractional differential equations with anti-periodic type boundary conditions. Open Math. 14, 723-735 (2016)

9. Klimek, M.: Sequential fractional differential equations with Hadamard derivative. Commun. Nonlinear Sci. Numer Simul. 16, 4689-4697 (2011)

10. Ye, H., Huang, R.: On the nonlinear fractional differential equations with Caputo sequential fractional derivative. Adv Math. Phys. 2015, Article ID 174156, 9 pp. (2015)

11. Alsaedi, A., Sivasundaram, S., Ahmad, B.: On the generalization of second order nonlinear anti-periodic boundary value problems. Nonlinear Stud. 16, 415-420 (2009)

12. Ahmad, B., Nieto, J.J.: Anti-periodic fractional boundary value problems. Comput. Math. Appl. 62, 1150-1156 (2011)

13. Ahmad, B., Losada, J., Nieto, J.J.: On antiperiodic nonlocal three-point boundary value problems for nonlinear fractional differential equations. Discrete Dyn. Nat. Soc. 2015, Article ID 973783, 7 pp. (2015)

14. Zhang, L., Ahmed, B., Wang, G.: Existence and approximation of positive solutions for nonlinear fractional integro-differential boundary value problems on an unbounded domain. Appl. Comput. Math. 15(2), 149-158 (2016)

15. Mahmudov, N.I., Unul, S.: On existence of BVP's for impulsive fractional differential equations. Adv. Differ. Equ. 2017, 15,16 pp. $(2017)$

16. Mahmudov, N.I., Unul, S.: Existence of solutions of $\alpha \in(2,3]$ order fractional three-point boundary value problems with integral conditions. Abstr. Appl. Anal. 2014, Article ID 198632 (2014)

17. Mahmudov, N.I., Bawaneh, S., Al-Khateeb, A.: On a coupled system of fractional differential equations with four point integral boundary conditions. Mathematics 7, 279 (2019)

18. Mahmudov, N.I., Al-Khateeb, A.: Stability, existence and uniqueness of boundary value problems for a coupled system of fractional differential equations. Mathematics 7, 354 (2019)

19. Huangi, Y., Liu, Z., Wang, R.: Quasilinearization for higher order impulsive fractional differential equations. Appl. Comput. Math. 15(2), 159-171 (2016)

20. Wang, J.R., Wei, W., Feckan, M.: Nonlocal Cauchy problems for fractional evolution equations involving Volterra-Fredholm type integral operators. Miskolc Math. Notes 13, 127-147 (2012)

21. Wang, J.R., Zhou, Y., Feckan, M.: On the nonlocal Cauchy problem for semilinear fractional order evolution equations. Cent. Eur. J. Math. 12, 911-922 (2104)

22. Ahmad, B., Ntouyas, S.K., Alsaedi, A.: On a coupled system of fractional differential equations with coupled nonloca and integral boundary conditions. Chaos Solitons Fractals 83, 234-241 (2016)

23. Henderson, J., Luca, R.: Nonexistence of positive solutions for a system of coupled fractional boundary value problems. Bound. Value Probl. 2015, 138 (2015)

24. Henderson, J., Luca, R., Tudorache, A.: On a system of fractional differential equations with coupled integral boundary conditions. Fract. Calc. Appl. Anal. 18, 361-386 (2015)

25. Wang, J.R., Zhang, Y.: Analysis of fractional order differential coupled systems. Math. Methods Appl. Sci. 38, 3322-3338 (2015)

26. Su, X.: Boundary value problem for a coupled system of nonlinear fractional differential equations. Appl. Math. Lett. 22, 64-69 (2009)

27. Hyers, D.H.: On the stability of the linear functional equation. Proc. Natl. Acad. Sci. USA 27(4), 222-224 (1941)

28. Jung, S.M.: On the Hyers-Ulam stability of the functional equations that have the quadratic property. J. Math. Anal. Appl. 222, 126-137 (1998)

29. Jung, S.M.: Hyers-Ulam stability of linear differential equations of first order II. Appl. Math. Lett. 19, 854-858 (2006)

30. Rassias, T.M.: On the stability of the linear mapping in Banach spaces. Proc. Am. Math. Soc. 72(2), $297-300$ (1978)

31. Benchohra, M., Lazreg, J.E.: On the stability of nonlinear implicit fractional differential equations. Matematiche 70(2), 49-61 (2015)

32. Castro, L.P., Simões, A.M.: Hyers-Ulam-Rassias stability of nonlinear integral equations through the Bielecki metric Math. Methods Appl. Sci. 41(17), 7367-7383 (2018)

33. Pending $\mathrm{Xu}$, L., Dong, Q., Li, G.: Existence and Hyers-Ulam stability for three-point boundary value problems with Riemann-Liouville fractional derivatives and integrals. Adv. Differ. Equ. 2018, Paper No. 458, 17 pp. (2018) 
34. Sousa, J.V.D.C., De Oliveira, E.C.: Ulam-Hyers stability of a nonlinear fractional Volterra integro-differential equation. Appl. Math. Lett. 81, 50-56 (2018)

35. Derakhshan, M.H., Ansari, A.: On Hyers-Ulam stability of fractional differential equations with Prabhakar derivatives. Analysis 38(1), 37-46 (2018)

36. Ali, A., Shah, K., Jarad, F.: Ulam-Hyers stability analysis to a class of nonlinear implicit impulsive fractional differential equations with three point boundary conditions. Adv. Differ. Equ. 2019(1), 7 (2019)

Submit your manuscript to a SpringerOpen ${ }^{\odot}$ journal and benefit from:

- Convenient online submission

- Rigorous peer review

- Open access: articles freely available online

- High visibility within the field

- Retaining the copyright to your article

Submit your next manuscript at $\boldsymbol{~ s p r i n g e r o p e n . c o m ~}$ 